\title{
Searching for the dead cone effects with iterative declustering of heavy-flavor jets
}

\author{
Leticia Cunqueiro ${ }^{1}$ and Mateusz Płoskoń ${ }^{2}$ \\ ${ }^{1}$ Oak Ridge National Laboratory, Physics Division, Oak Ridge, 37831 Tennessee, USA \\ ${ }^{2}$ Lawrence Berkeley National Laboratory, 1 Cyclotron Rd, Berkeley, 94720 California, USA
}

(Received 28 February 2019; published 26 April 2019)

\begin{abstract}
We present a new method to expose the dead-cone effect at colliders using iterative declustering techniques. Iterative declustering allows one to unwind the jet clustering and to access the subjets or branches at different depths of the jet tree. Our method consists of declustering the heavy flavor-tagged jet using the Cambridge-Achen algorithm following the branch containing the heavy flavor at each step and registering the kinematics of the complementary untagged prong. The kinematics of the complementary untagged prong fill a Lund map representing the gluon radiation off the heavy-flavor quark at each step of the vacuum shower. Using PyTHIA8 Monte Carlo, we show that a simple cut on the Lund plane introduced by $\ln \left(k_{\mathrm{T}}\right)>0$ suppresses hadronization effects and the angular separation between the jet prongs becomes very sensitive to flavor effects. A clear suppression for heavy-flavor jets relative to inclusive jets in the region of splitting angles delimited by the relation $\theta<m_{Q} / E$ is observed, where $m_{Q}$ is the mass of the heavy quark and $E$ is the energy of the radiator or splitting prong.
\end{abstract}

DOI: 10.1103/PhysRevD.99.074027

\section{INTRODUCTION}

The dead-cone effect is a fundamental prediction of QCD (and gauge theories in general) according to which the radiation from a charged particle of mass $m$ and energy $\mathrm{E}$ is suppressed at angular scales given by $\mathrm{m} / \mathrm{E}[1]$.

To experimentally uncover the dead cone is a difficult task. The decays of the heavy-flavor particles happen at similar angular scales and fill the dead cone. The irresolution of the axis chosen as proxy for the heavy-flavor direction can also obscure its measurement. In Ref. [2], the first direct measurement in $\mathrm{e}^{+} \mathrm{e}^{-}$collisions of the depletion of fragmentation particles not coming from the heavyflavor decay vertex in heavy-flavor tagged jets was presented. The main limiting element was the choice of the reference axis, which was estimated using the thrust direction, the jet axis, and the vertex direction.

Recently, new ideas were proposed to measure the dead cone using SOFT DROP grooming techniques and boosted top quarks at the Large Hadron Collider [3].

In this paper, we discuss the use of new iterative declustering techniques $[4,5]$ that allow one to penetrate the jet shower and access the deepest levels of the clustering history which correspond to the splittings at the smallest

Published by the American Physical Society under the terms of the Creative Commons Attribution 4.0 International license. Further distribution of this work must maintain attribution to the author(s) and the published article's title, journal citation, and DOI. Funded by SCOAP ${ }^{3}$. angles. This may allow one to unveil the dead cone even for low mass heavy flavors such as charm and beauty, provided they can be fully reconstructed in the experiment and that the angular resolution of the detector is good enough to separate subjets at distances of order $0.1 \mathrm{rad}$, which is typically the case with a tracking device.

\section{ITERATIVE DECLUSTERING OF HEAVY FLAVOR-TAGGED JETS}

We have generated PyTHIA8.226 Tune 4C [6] hard events leading to charm and beauty quark-initiated jets, and we have inhibited the decay of the $\mathrm{D}$ and $\mathrm{B}$ mesons. We reconstruct the jets with the anti- $\mathrm{k}_{\mathrm{T}}$ algorithm [7] and tag the jets when a $\mathrm{D}^{0}$ or $\mathrm{B}^{0}$ meson is found as one of its constituents. The jet resolution parameter is $R=0.4$, the E-scheme recombination is used, and no minimum particle transverse momentum cutoff is considered.

The tagged jets are then declustered using the Cambridge/Aachen (C/A) algorithm [8]. Since the C/A metric is such that particles at small angles are combined first, the first steps of the declustering process find the prongs at the largest relative angles.

When moving backward through the jet history, we always follow the branch containing the heavy flavor, and we register the relative transverse momentum $k_{\mathrm{T}}$ and angle $\theta$ of the complementary untagged prong onto the Lund map, where $k_{\mathrm{T}}=\omega \sin (\theta)$ and $\omega$ is the energy carried by the complementary prong. The cases where the heavyflavor tagged prong is not the hardest are of the orders of 
$1 \%$ and $0.001 \%$ in the explored kinematic regime for $\mathrm{c}$ and $\mathrm{b}$ jets, respectively, and their exclusion from the Lund map has no quantitative impact on the results, so there are no ambiguities in the comparison to the inclusive jets, in which case we follow the hardest prong.

Figure 1, left column, shows the Lund maps for b- and c-tagged jets together with inclusive jets, at parton level. The $y$ and $x$ axes are $\ln \left(k_{\mathrm{T}}\right)$ and $\ln (1 / \theta)$, respectively.
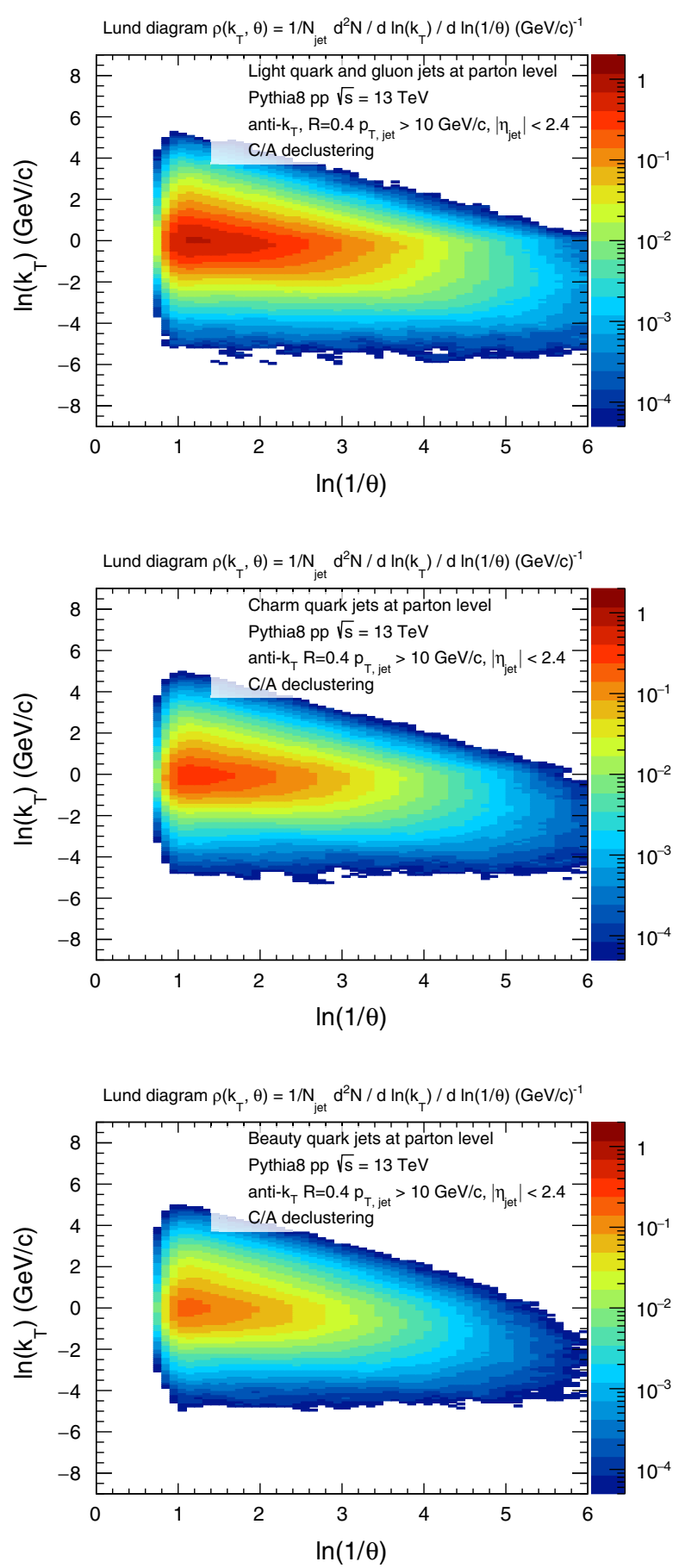

The two-dimensional (2D) maps are normalized to the total number of jets. The underlying event was switched off.

Already visually, without further analysis, one can note that the small-angle region is less filled for heavy quarks jets than for inclusive jets.

Figure 1, right column, shows the Lund maps for b- and c-tagged jets together with inclusive jets at hadron level. The hadronization effects pollute the low $\ln \left(k_{\mathrm{T}}\right)$ part of the
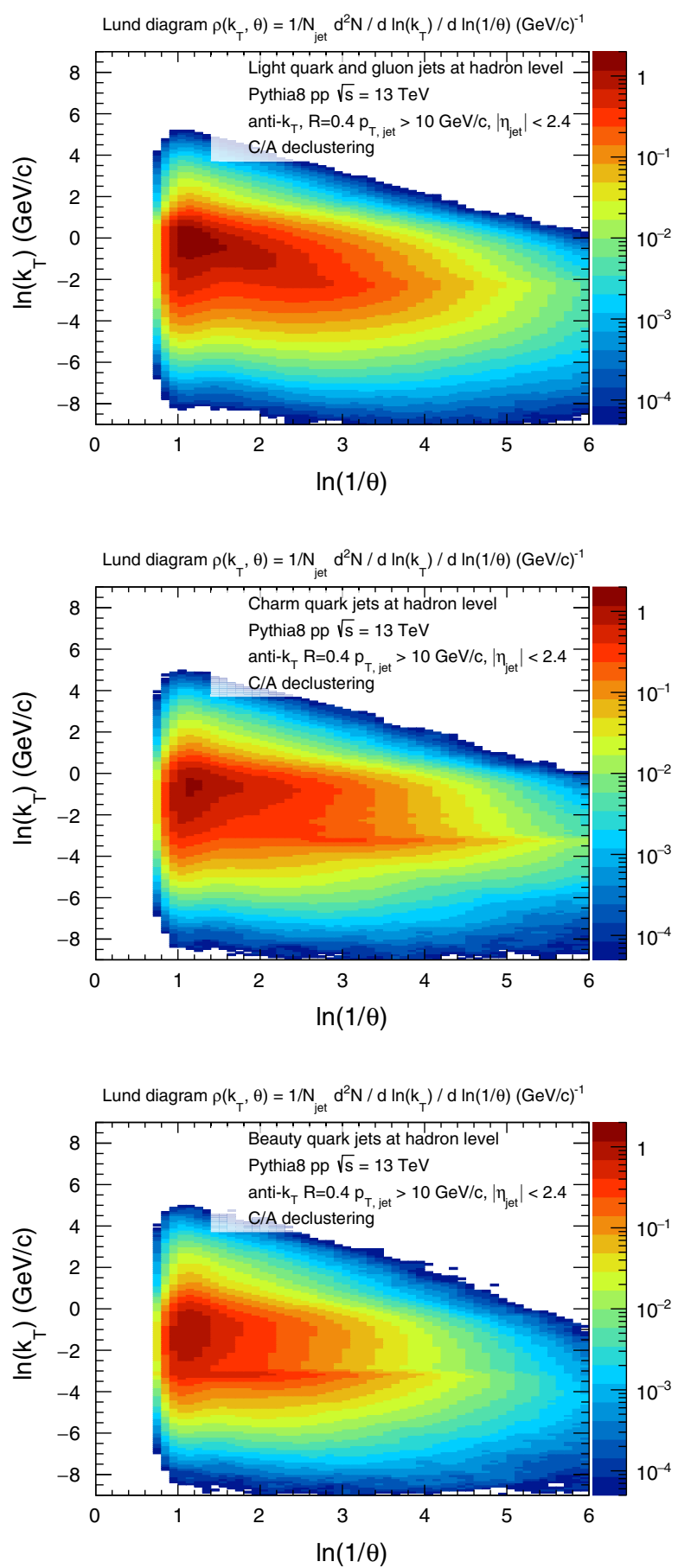

FIG. 1. Left column: Lund diagrams for charm and beauty and inclusive jets at parton level and underlying event switched off, for low momentum jets of $10<p_{T \text {,jet }}<40$. Right column: The diagrams at hadron level with underlying event included. 

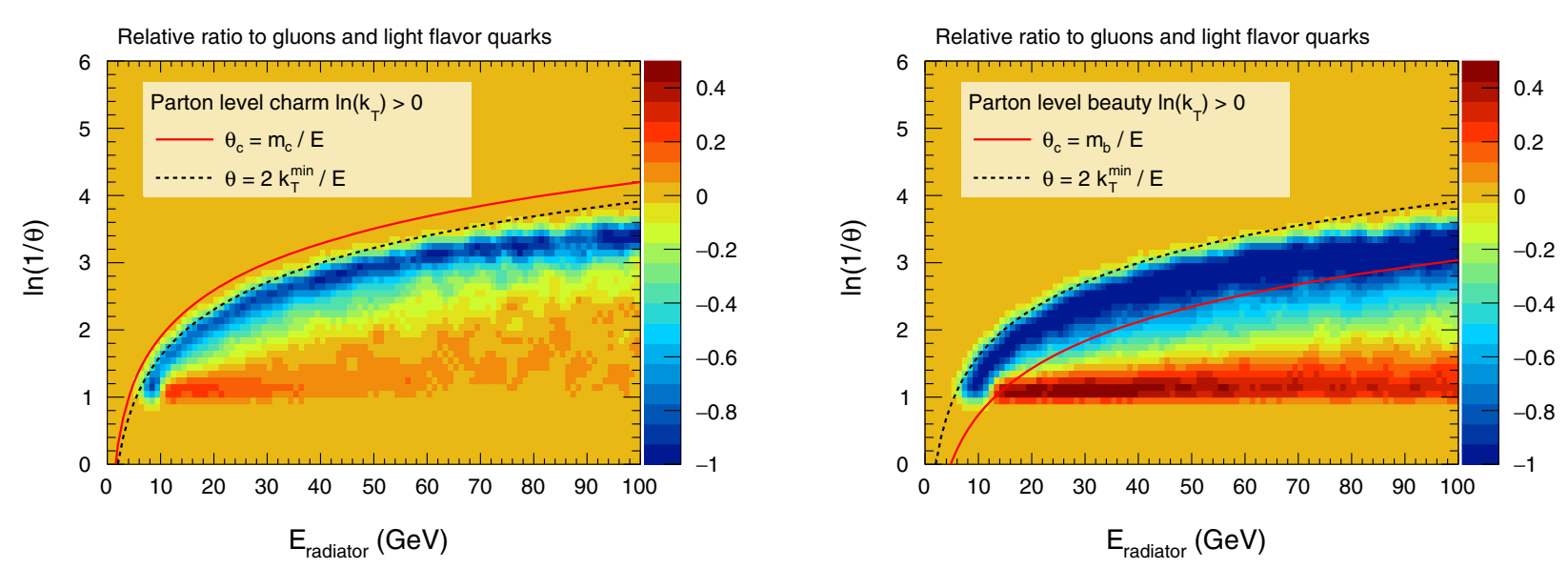

(a)
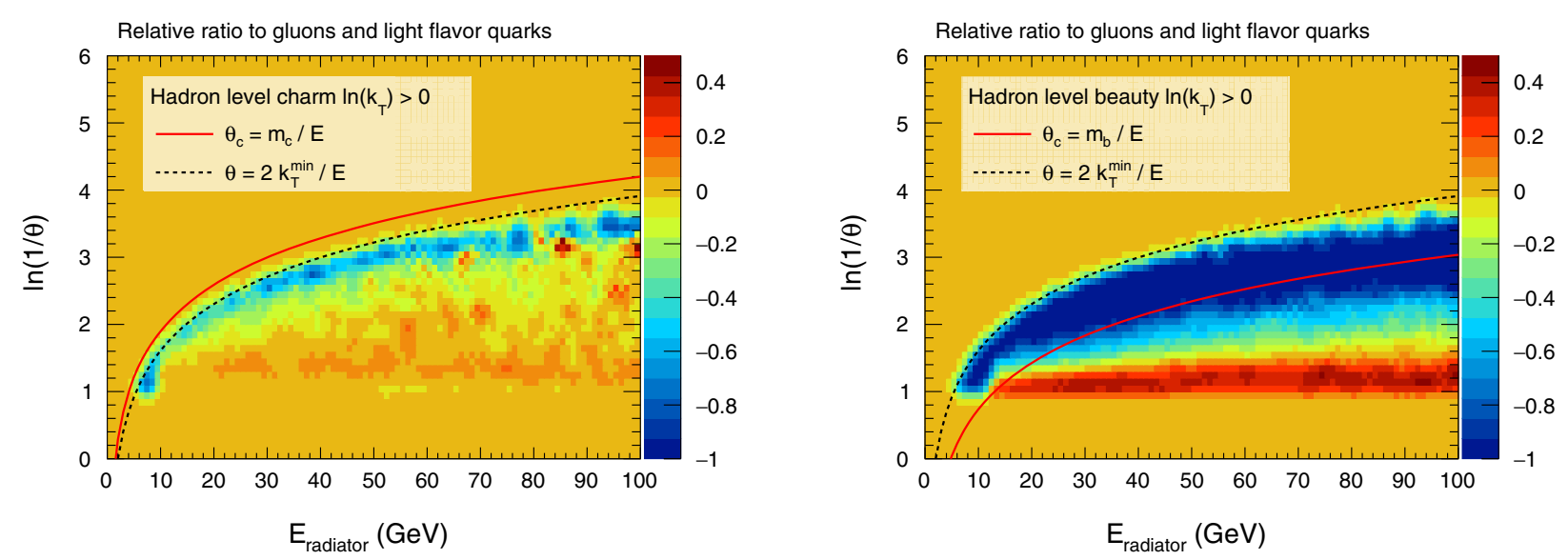

(b)
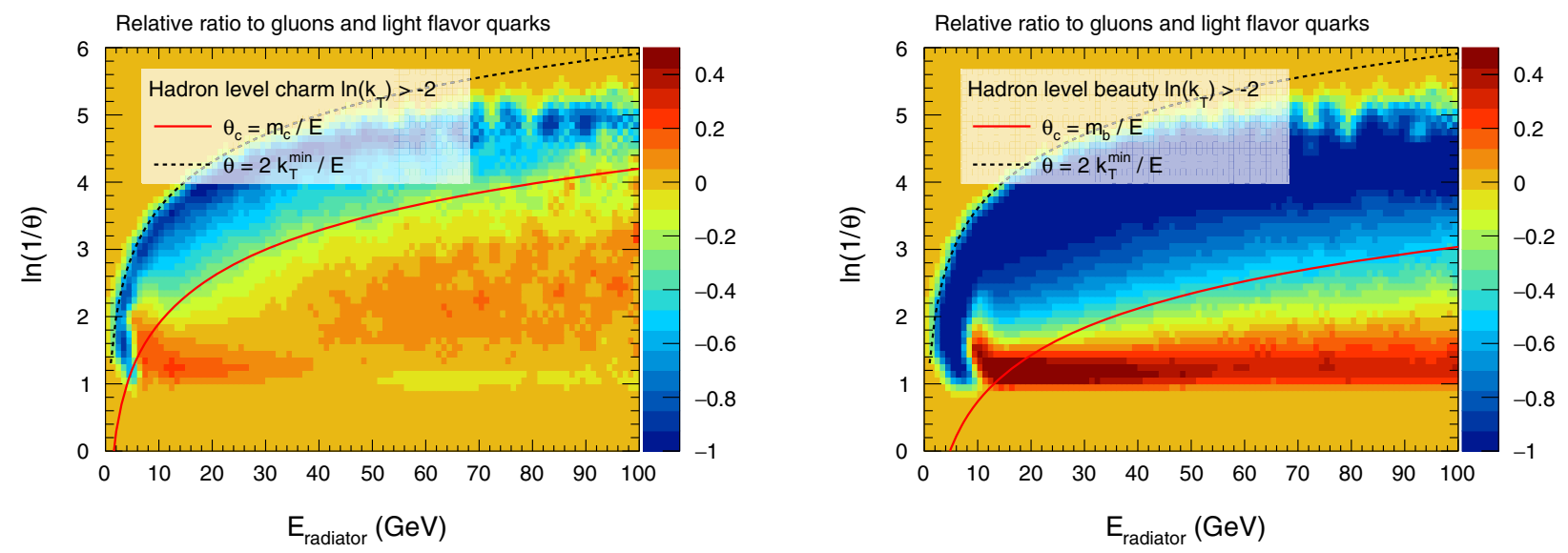

(c)

FIG. 2. Relative difference for heavy-flavor and inclusive jets of the correlation of the splitting angle and the energy of the radiator. The red curves correspond to the critical angle $\theta_{C}=m_{Q} / E$. (a) Parton level c-jets (left) and b-jets (right). (b) Hadron level c-jets (left) and b-jets (right). (c) Hadron level with a relaxed cut on $k_{\mathrm{T}}$-demonstration of the impact of non-perturbative effects.

diagram, which corresponds to nonperturbative scales. The underlying event pollutes the large-angle sector, where the catchment area is maximal. In order to suppress nonperturbative effects, a simple cut on the splitting scale is possible, $\ln \left(k_{\mathrm{T}}\right)>0$, which selects splittings governed by scales of at least $1 \mathrm{GeV} / c$. 


\section{DEAD CONE}

A different representation of the Lund diagram which exposes the dead-cone effect more clearly is that where the horizontal axis corresponds to the energy of the radiating daughter prong and the vertical axis corresponds to the splitting angle $\theta$. We construct this 2D map, and we consider the relative difference between heavy quarktagged jets and inclusive jets as a way to study the relative enhancement/suppression in the different areas of interest,

$$
Q=\frac{P^{Q}\left(\ln (1 / \theta), E_{\text {radiator }}\right)-P^{\text {inc }}\left(\ln (1 / \theta), E_{\text {radiator }}\right)}{P^{\text {inc }}\left(\ln (1 / \theta), E_{\text {radiator }}\right)},
$$

where $P\left(\ln (1 / \theta), E_{\text {radiator }}\right)$ represents the probability for a radiator prong with energy $E_{\text {radiator }}$ to split with an aperture angle $\theta$. This is illustrated in Fig. 2(a). The cut $\ln \left(k_{\mathrm{T}}\right)>0$ translates into $E_{\text {radiator }}>1 /(z \theta)$, where $z$ is the energy fraction carried by the daughter prong. The kinematic limit $z=0.5$ imposes the sharp threshold in the curve above which there are no entries in the inclusive reference.

One can clearly see a region of the phase space where $Q$ becomes negative, indicating a suppression of the splittings for the heavy quarks compared to inclusive jets, both for c (left) and b jets (right). The angular suppression is coupled to a suppression of the high $z$ splittings: the suppression of small angles affects hard emissions with small $k_{\mathrm{T}}$, which are the most probable. This leads to a reduction of the intrajet multiplicity and to a hardening of the fraction of energy kept by the leading prong in the jet.

The region of angles smaller than $\theta<\theta_{C}=m_{Q} / E$ corresponds to the area above the red curve. We note that the parametrical dead-cone red line qualitatively coincides with the curve where $Q_{\text {Beauty }}$ becomes -1 , when radiation of the heavy-flavor prong is completely suppressed, though the relation is not exact. In the case of charm, the dead-cone line is above the kinematic threshold for the given $k_{\mathrm{T}}$ selection. More aggressive cuts on the scale $k_{\mathrm{T}}$ select more

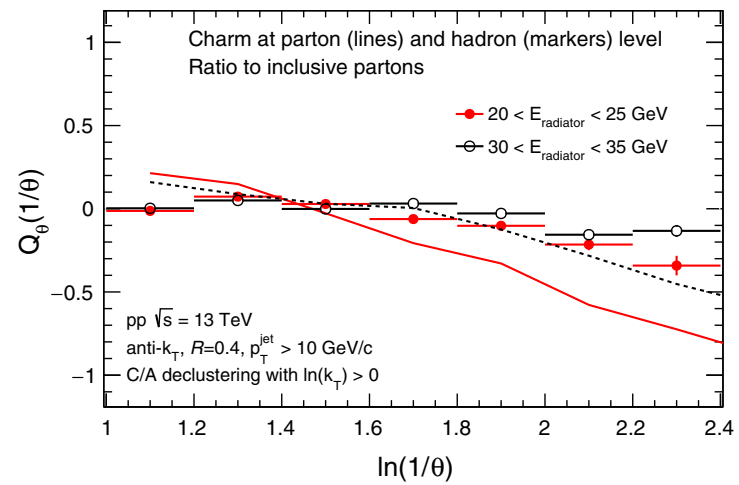

large-angle radiation and reduce the range of observation of the dead-cone related suppression.

At hadron level, the effects are not washed out. A suppression of significant magnitude at angles smaller than the critical angle at all radiator energies for $\mathrm{b}$ and $\mathrm{c}$ jets is observed; see Fig. 2(b).

In Fig. 2(c), we show the impact of a looser cut $\ln \left(k_{\mathrm{T}}>-2\right)$. Nonperturbative effects fill the dead cone and obscure the effect of the suppression of the radiation. In Fig. 3, we show our proposed observable, which is projection onto the vertical axis of the diagrams shown in Fig. 2(b), for a range of low radiator energies, for instance $20<E_{\text {radiator }}<50 \mathrm{GeV}$. The observable, denoted as $Q_{\theta}$, corresponds to the relative difference between the angular distribution of the splittings for heavy-flavor jets and inclusive jets, at perturbative scales set by $\ln \left(k_{\mathrm{T}}\right)>0$ and for low radiator energies for which the dead-cone effects are maximal:

$$
Q_{\theta}=\frac{P^{Q}(1 / \theta)-P^{\text {inc }}(1 / \theta)}{P^{\text {inc }}(1 / \theta)}, \quad E_{\text {radiator }} \in\left(E_{\min }, E_{\max }\right) .
$$

The suppression of the low-angle emission probability for b-tagged radiators relative to inclusive ones is of order $80 \%$ at $\ln (1 / \theta)=2$, which approximately corresponds $0.14 \mathrm{rad}$. The corresponding suppression for c-tagged radiators is of order $20 \%$.

The ideal experiment would be able to fully reconstruct the heavy-flavor hadrons and to tag prongs of very low momentum and to separate subjets at angular scales of 0.1 and below.

We note that the inclusive jets are not the best reference since they are a mixture of $g \rightarrow g g$ and $q \rightarrow q g$ radiation with different fractions. The $g \rightarrow g g$ fill the phase space in a different way compared to $q \rightarrow q g$. We have checked that the magnitude of the suppression of $Q_{\theta}$ increases when light quark jets are considered as a reference instead of inclusive jets; see Fig. 4. Experimentally, it is possible to

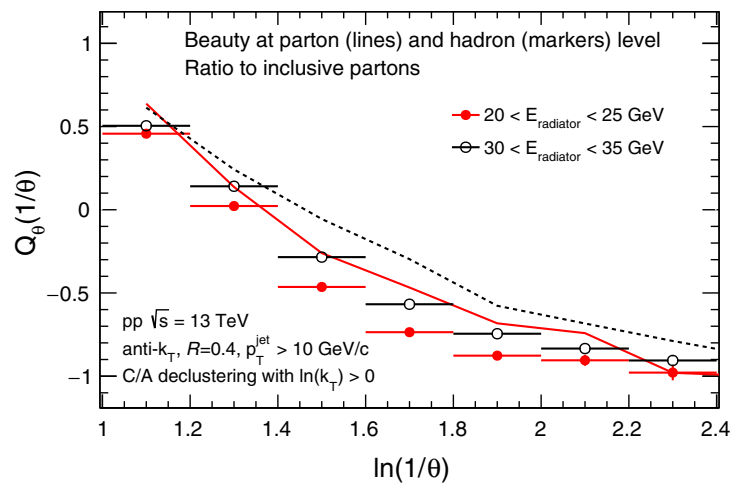

FIG. 3. Relative difference of the angular distribution for heavy-flavor and inclusive jets, for perturbative scales defined by $\ln \left(k_{\mathrm{T}}>0\right)$, and for different bins of radiator energy. Left and right plots correspond to charm and beauty jets, while solid/dashed lines correspond to hadron/parton level. 

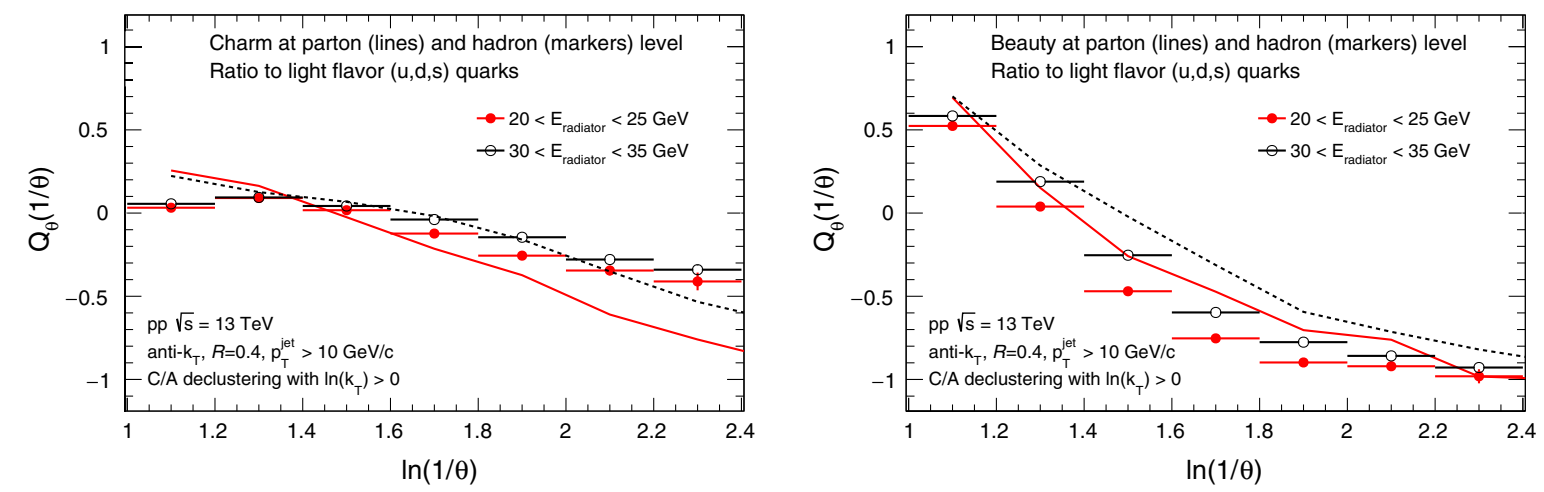

FIG. 4. Relative difference of the angular distribution for heavy-flavor and light quark jets, for perturbative scales defined by $\ln \left(k_{\mathrm{T}}>0\right)$ and for different bins of radiator energy. Left and right plots correspond to charm and beauty jets, while solid/dashed lines correspond to hadron/parton level.

enrich the quark fraction by using boson-jet correlations for instance or by statistically cutting on observables that are sensitive to differences between quark and gluon fragmentation such as the $p_{T} D$ or the jet angularity.

We have also tested that switching on/off the gluon splitting kernel $g \rightarrow q q b a r$ has no impact on the results.

For a final remark, we note that our method was tested only against PYTHIA8 and thus the exact magnitude of the dead-cone related effects and their onset rely on the specific PYTHIA8 implementation, which is done via matrix element corrections. However, parton shower generators feature the dead-cone effect quite universally with better than $10 \%$ agreement with next-to-leading-order calculations [3].

\section{CONCLUSIONS}

We have shown that iterative declustering is a tool that allows us to access the deepest branches of the C/A jet trees which correspond to the smallest splitting angles and that can uncover quark mass differences related to the dead cone. We propose to build the Lund map for jets tagged with a fully reconstructed heavy-flavor hadron. Furthermore, we propose to expose the perturbative splittings within the shower with a selection of $\ln \left(k_{\mathrm{T}}\right)>0$ and study the angular effects due to the dead cone at low radiator energies integrating over all declustering steps. At angles of order $0.1 \mathrm{rad}$, the PYTHIA8 simulations predict a suppression of approximately $20 \% / 80 \%$ for jets containing a fully reconstructed $\mathrm{D} / \mathrm{B}$ meson relative to inclusive jets, for radiator energies above $20 \mathrm{GeV}$.

\section{ACKNOWLEDGMENTS}

The authors thank Gavin Salam and Marco van Leeuwen for useful discussions and comments on the manuscript. This work was supported by the U.S. Department of Energy, Office of Science, Office of Nuclear Physics, under Contracts No. DE-AC05-00OR22725 (ORNL) and No. DE-AC02-05CH11231 (LBNL).
[1] Y. L. Dokshitzer, V. A. Khoze, and S. I. Troian, On specific QCD properties of heavy quark fragmentation ('dead cone'), J. Phys. G 17, 1602 (1991).

[2] M. Battaglia, R. Orava, and L. Salmi, A study of depletion of fragmentation particles at small angles in b-jets with the DELPHI detector at LEP, CERN Report No. DELPHI-2004037 CONF 712, 2004.

[3] F. Maltoni, M. Selvaggi, and J. Thaler, Exposing the dead cone effect with jet substructure techniques, Phys. Rev. D 94, 054015 (2016).

[4] F. A. Dreyer, G. P. Salam, and G. Soyez, The Lund jet plane, J. High Energy Phys. 12 (2018) 064.
[5] H. A. Andrews et al., Novel tools and observables for jet physics in heavy-ion collisions, arXiv:1808.03689.

[6] T. Sjöstrand, S. Ask, J. R. Christiansen, R. Corke, N. Desai, P. Ilten, S. Mrenna, S. Prestel, C. O. Rasmussen, and P. Z. Skands, An introduction to PYTHIA 8.2, Comput. Phys. Commun. 191, 159 (2015).

[7] M. Cacciari, G. P. Salam, and G. Soyez, The anti- $k_{t}$ jet clustering algorithm, J. High Energy Phys. 04 (2008) 063.

[8] Y. L. Dokshitzer, G. D. Leder, S. Moretti, and B. R. Webber, Better jet clustering algorithms, J. High Energy Phys. 08 (1997) 001. 